\section{P253 ISONIAZID AND MULTI-DRUG RESISTANT MYCOBACTERIUM TUBERCULOSIS: THE EAST LONDON EXPERIENCE}

H Liddicoat, S Mohd-Afzal, J Potter, V White, N Jayasekera, M Darmalingam, H Kunst. Barts Health NHS Trust, London, UK

\subsection{6/thoraxjnl-2015-207770.389}

Introduction East London has one of the UK's largest cohorts of Drug Resistant Tuberculosis (DR-TB). This paper aims to provide insight into the recent behaviour of DR-TB in a multi-ethnic urban TB clinic.

Methods A retrospective study was conducted on all patients with DR-TB between 2007 and 2013. Statistical analysis was performed using Fisher's Exact Test.

Results 179 cases were identified: 126 patients had Isoniazid mono-resistance (H-Mono), 3 had poly-resistance including Isoniazid, and 37 patients had multi-drug resistant TB (MDR-TB). There were 6 cases of Rifampicin mono-resistance, 1 Ethambutol mono-resistance, and 6 Pyrazinamide mono-resistance.

H-Mono and MDR-TB were more prevalent in males $(58 \%$ and $57 \%$ respectively). H-Mono predominated in younger age groups (mode age group 25-29) whilst MDR-TB had a more uniform age distribution (Figure 1). The ethnicities of patients with DR-TB reflected the local population (Indian Subcontinent: 57\% of H-Mono and 52\% MDR-TB; Africa: 16\% for both; Caucasian: $11 \%$ and 16\% respectively; Other: $16 \%$ for both).

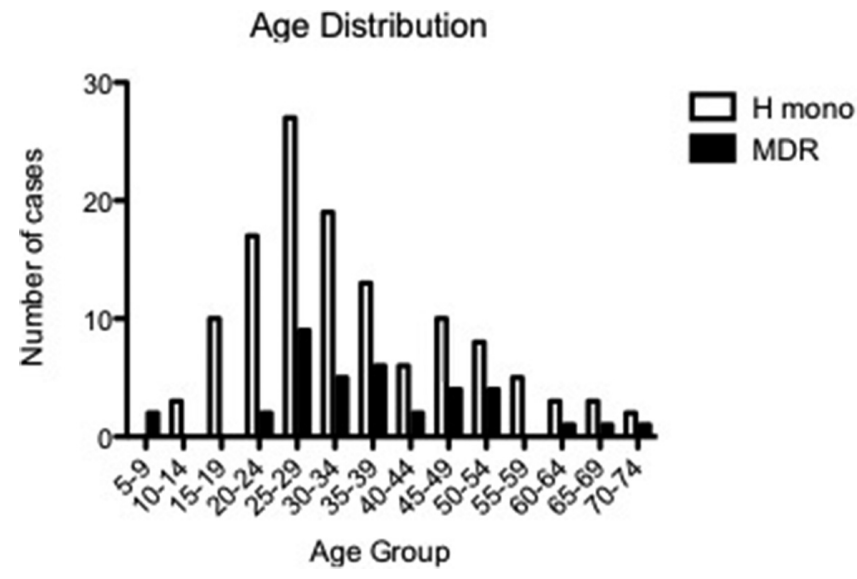

Abstract P253 Figure 1

The majority were non-UK born, however a meaningful proportion were UK-born (H-Mono 17\% and MDR-TB 14\%). Patients from all WHO world regions with DR-TB were more likely to have H-Mono, apart from Eastern Europe, where MDR-TB was more common ( $\mathrm{p}=0.005)$.

The proportion of patients with pulmonary TB was similar in both cohorts (54\% H-Mono and 65\% MDR-TB, $\mathrm{p}=0.26)$, as was the proportion of patients who were sputum smear-positive (46\% and $38 \%$ respectively, $p=0.64$ ).

More patients with MDR-TB had a history of previous TB diagnosis compared to patients with H-Mono $(p=0.0005)$.

Of note, the 5 UK-born patients with MDR-TB were all under the age of 40 with no specific risk factors or previous TB treatment.

The number of TB notifications fell during this period, however the proportion of patients with MDR-TB remained stable at approximately $1 \%$. In contrast, cases with any drug resistance reduced.
Conclusion H-mono and MDR-TB shared many demographical features, but in this cohort there were significant differences in age distribution, previous diagnosis of TB and country of origin. A significant proportion of patients did not match the typical profile of DR-TB, highlighting the importance of culture to exclude drug-resistance in all individuals.

\section{P254 UTILISING COMMUNITY EMPOWERMENT AND BIOMETRICS TO IMPROVE TUBERCULOSIS TREATMENT OUTCOMES IN DELHI'S SLUM POPULATION: THE OP ASHA MODEL}

${ }^{1} \mathrm{D}$ Pan, ${ }^{2} \mathrm{E}$ Lee, ${ }^{2} \mathrm{~L}$ Lock, ${ }^{3} \mathrm{R}$ Batra, ${ }^{4} \mathrm{I}$ Abubakar, ${ }^{3} \mathrm{~S}$ Batra, ${ }^{5} \mathrm{M}$ Lipman. ${ }^{1}$ Imperial College School of Medicine, London, UK; ${ }^{2}$ Wellesley College, Massachusetts, USA; ${ }^{3}$ Operation ASHA, Delhi, India; ${ }^{4}$ University College London, London, UK; ${ }^{5}$ Royal Free Hospital, London, UK

\subsection{6/thoraxjnl-2015-207770.390}

Introduction India accounts for more than one-fifth of the total global tuberculosis (TB) incidence. In response, the Revised National TB Control Program (RNTCP) of India has supported the decentralisation of service delivery with encouragement of active community participation. Operation ASHA is an international NGO that offers TB services to slum populations - a group that have proven difficult historically to engage. It employs a unique treatment approach involving communitybased providers and biometric data registration to support adherence. We report treatment outcome for patients with presumed drug-susceptible tuberculosis managed by Op ASHA within Delhi's slum districts.

Methods Retrospective, observational cohort study focusing on patients within 14 southern Delhi slum districts started on treatment according to RNTCP guidelines between 1.4.2012 and 31.3.2013. Demographic and clinical information were systematically recorded. Patients still on treatment by the end of October 2013 were excluded from analysis. Successful outcomes were defined as bacteriologically confirmed cure or treatment completion. Adverse outcomes were: treatment default, conversion to MDR-TB treatment, treatment failure or death.

Results 1217 patients were started on treatment. 29\% were aged $<20$; and $42 \%$ were female. $52 \%$ had pulmonary $\mathrm{TB}$; of these $55 \%$ were sputum smear positive $(58 \%$ grade II or III out of III). 218 (18\%) subjects re-treated for TB were excluded from further outcome analysis. The remaining 999 patients had similar clinical characteristics to the whole study population. Successful outcome after 6 months of treatment was recorded in $85 \%$ of subjects $(77 \%$ pulmonary, $91 \%$ extrapulmonary cases). Adverse outcomes were recorded in $16 \%$ of those with pulmonary TB. This was considerably less common in extrapulmonary $\mathrm{TB}$, where it was recorded in only $6 \%$. Death was reported in $3 \%$ of pulmonary and $1 \%$ of extrapulmonary patients.

Conclusions The model of care achieves the WHO target for treatment completion of $85 \%$ in a population considered very high risk of failure under traditional TB programmes. Future work needs to focus on the factors that result in adverse outcomes, in particular for the key patient population with infectious pulmonary $\mathrm{TB}$. 